\title{
Mild head injury in preschool children: evidence that it can be associated with a persisting cognitive defect
}

\author{
Philip Wrightson, Valerie McGinn, Dorothy Gronwall
}

\begin{abstract}
This study describes the effect of mild head injury in preschool children on aspects of their cognitive performance in the year after injury and at the age of 6.5 years, with particular reference to the development of reading skills. Mild head injury was defined by diagnosis at a hospital emergency department of a head injury which was not severe enough to need admission for observation. Seventy eight such children were compared with a group of 86 with a minor injury elsewhere. The groups had similar developmental, family, and socioeconomic status. There were no differences in cognitive tests soon after the injury, but at six months and one year children with mild head injury scored less than controls on one test, solving a visual puzzle (visual closure); they were also more likely to have had another mild head injury. At $6 \cdot 5$ years of age they still scored less than controls, reading ability was related to their visual closure score at one year, and they were more likely to have needed help with reading.

Mild head injury seems to be able to produce subtle but significant changes which can affect school performance.
\end{abstract}

(F Neurol Neurosurg Psychiatry 1995;59:375-380)

Keywords: preschool children; head injury; cognitive impairment

Some children who have a mild head injury do badly at school afterwards, either because of their behaviour or because they cannot cope with the school work. Several studies have suggested that the head injury is not responsible. One view is that the sort of children who are injured are more likely to have had behaviour problems before the injury. ${ }^{12}$ Another is that although the children may show defects of cognitive function, this is not proportional to the apparent severity of the injury, and that the changes do not show the pattern of loss and subsequent recovery typical of CNS damage, so that it is more likely that the deficit was there before the injury. ${ }^{3} \mathrm{~A}$ long term follow up of children with various injuries suggested that cognitive changes were common in all, and that the children with head injuries were not specially affected. ${ }^{4}$ It has also been suggested that parental overreaction and family dysfunction may be a factor. ${ }^{5}$

Our previous work had shown that in adults the defects of cognitive function that follow a mild head injury can persist for many months, ${ }^{67}$ and we saw no reason why children should be different. In 1982, prompted by suggestions from teachers and parents that young children who had had head injuries were slow in learning to read, a pilot study was completed comparing 10 preschool children with mild head injury with 10 matched for the factors known to affect learning. No difference between the groups was found immediately after the injury, but eight months later the children with head injury were less able to complete visual puzzles. It seemed reasonable to suppose that children who found this difficult might have problems in learning to read, and further investigation of this area was clearly warranted.

There are, however, practical problems in showing changes of cognitive function and their relation to injury in preschool children. Assessment of very young children is difficult. It may be complicated by factors such as shyness, distractability, and developmental delay, which are not related to the accident. Further, they belong to a group in which information on academic achievement is absent, so that the child's capacity before the head injury cannot be estimated. On the other hand the family and socioeconomic factors which affect learning at this age have been well documented and can be allowed for. Also, as this is a period of intense learning activity loss of capacity might be expected to produce greater effects than in older children.

A more rigorous investigation was therefore planned, with the hypothesis that mild head injury in preschool children would affect their learning to read. The development of this skill has been well studied and quantified, and would provide a good endpoint. The children would be followed up for a year from the date of their accident, and then seen again at the age of 6.5 years when their school progress and particularly their reading skills would be assessed.

\section{Subjects and methods} SUBJECTS

Consecutive names were taken from the records of two general hospitals of children who had attended the accident departments and had been diagnosed as having had either a mild head injury (HI group) or a minor injury to another part of the body (control group). Within a week of the accident the parents were telephoned and their consent to enter the 
children into the study sought. The child was included providing: (1) that hospital admission had not been needed; (2) that the child was between 2.5 and 4.5 years old; (3) that there was no history of previous head injury or another condition which might confuse the issue; and (4) that the first language spoken at home was English.

Eighty nine children were identified as possible subjects for each group (HI and control). 11 of the HI group and three of the controls had then to be excluded because of a previous head injury, leaving 78 in the $\mathrm{HI}$ group and 86 controls.

As the investigation progressed head injuries occurred in both the HI group and the controls. These children continued to be examined but data obtained after the further injury was not used for the present analysis.

\section{METHODS}

Part 1

Each child was seen within a month of the injury, then six and 12 months later.

Assessments were all made in the child's home, with the help of a parent. The first was done as soon as the parent considered that the child was well enough to be tested, usually within 14 days of the accident. The other assessments were done six and 12 months later. Two sessions of an hour were needed for each, both being completed within a week. The following information was obtained:

Clinical history-The parents were asked for the details of the accident, the immediate symptoms, and the child's progress afterwards. Note was taken of previous illnesses or accidents which might affect development. At the second and third visits the occurrence of subsequent injuries, to the head or elsewhere, was recorded.

Family and socioeconomic status-The ages and education of the parents, the number and age of the siblings, and the child's position in the family were noted. A measure of socioeconomic status designed for use in New Zealand was recorded. ${ }^{8}$

Behavioural measures-The Vineland social maturity scale ${ }^{9}$ and the Connors parent questionnaire ${ }^{10}$ were used to assess social maturity and behaviour.

Cognitive tests - The Reynell developmental language scales ${ }^{11}$ were used as a broad measure of expressive and receptive language ability. The battery uses a collection of attractive toys and everyday objects, and is designed for children from 1 to 5 years old.

The Illinois test of psycholinguistic abilities (ITPA), ${ }^{12}$ which is suitable for children from 2.5 to 10 years old, was used to provide an assessment of a wide range of language and related cognitive skills. Its 10 subtests are:

(1) Auditory reception: deriving meaning from spoken questions such as "Do cats bark?"

(2) Visual reception: finding the conceptually related response picture when presented with a stimulus picture.

(3) Visual sequential memory: reproducing sequences of geometric figures from memory after looking at them for five seconds.

(4) Auditory association: completing sentences such as "I sit on a chair, I sleep on a -".

(5) Auditory memory: remembering sequences of digits.

(6) Visual association: finding a response picture which goes with a stimulus picture.

(7) Visual closure: finding partially concealed objects embedded in pictures within a time limit of thirty seconds.

(8) Verbal expression: describing common objects.

(9) Grammatical closure: using appropriate grammar to complete sentences in response to pictures.

(10) Manual expression: miming the use of common objects.

Part 2

The children were seen again when they reached the age of 6.5 years. They were tested on two occasions, once at home with a parent present, and once at school with the cooperation of their teacher. The following tests were carried out:

In the first session, at home, the Illinois test of psycholinguistic abilities, the Connors parent behaviour questionnaire, and the Vineland social maturity scale were given to assess the child's present capacity and to document change since the previous tests. Parents were asked about general progress and whether there had been further head injuries.

In the second session, at school, the tests presented were:

(1) The Neale analysis of reading ability: ${ }^{13}$ a graded series of illustrated stories with comprehension questions.

(2) Letter knowledge and writing: the child is asked to say the name and the sound of lower and upper case letters of the alphabet and asked to write six words.

(3) Coding subtest of the Wechsler intelligence scale for children: this is a timed attention task.

(4) Verbal memory passage: this is a measure of immediate and delayed recall using a passage suitable to the child's age.

(5) Visual memory test: immediate memory of four geometric figures adapted from the Wechsler memory scale to be suitable for children.

(6) Paired associate learning: a measure of ability to learn verbal material adapted from the Wechsler memory scale.

(7) Frostig developmental test of visual perception: tasks to assess various perceptual skills. ${ }^{14}$

(8) Connors teacher's questionnaire: the teacher rates the child on items concerning its behaviour at school. It was also noted whether problems with reading which required special tuition had been recognised by the teacher.

STATISTICAL METHODS

The methods used are given with each finding. In analysing the results of the cognitive tests the raw scores were used as dependent variables. This allowed direct comparison of indi- 
vidual subjects over time, and avoided the uncertainty of using scores scaled for age some years before and in a different population.

\section{ETHICAL ISSUES}

Both parts of the study were approved by the Auckland Hospital ethics committee. The parents were told in detail about the aims of the investigation before being asked for consent. In the second part informed consent was also obtained from both the Auckland Education Board and the principals of the schools visited.

\section{Results \\ PART 1 \\ Comparability of groups}

There was no significant difference between the groups in sex, pre-accident development and behaviour, socioeconomic state, or mother's or father's age or education levels. In the subjects available for study at one year there was a slightly higher proportion of younger children in the $\mathrm{HI}$ group, the mean and median ages being 3.38 and 3.25 years

Visual closure scores immediately after injury, six and 12 months later, and at the age of $6 \cdot 5$ years

\begin{tabular}{lccccc}
\hline & \multicolumn{2}{l}{ Mean scores } & & \multicolumn{2}{l}{ Anova } \\
\cline { 2 - 3 } \cline { 5 - 6 } Time & Head injury & Control & & Fvalue & P value \\
\hline First & $4 \cdot 79$ & $5 \cdot 11$ & & 0.02 & 0.89 \\
Six months & 5.47 & $7 \cdot 00$ & & 20.43 & 0.0001 \\
12 months & 6.60 & 8.42 & & 10.61 & 0.0016 \\
Age 6.5 years & 22.44 & 25.70 & 6.19 & 0.015 \\
\hline
\end{tabular}
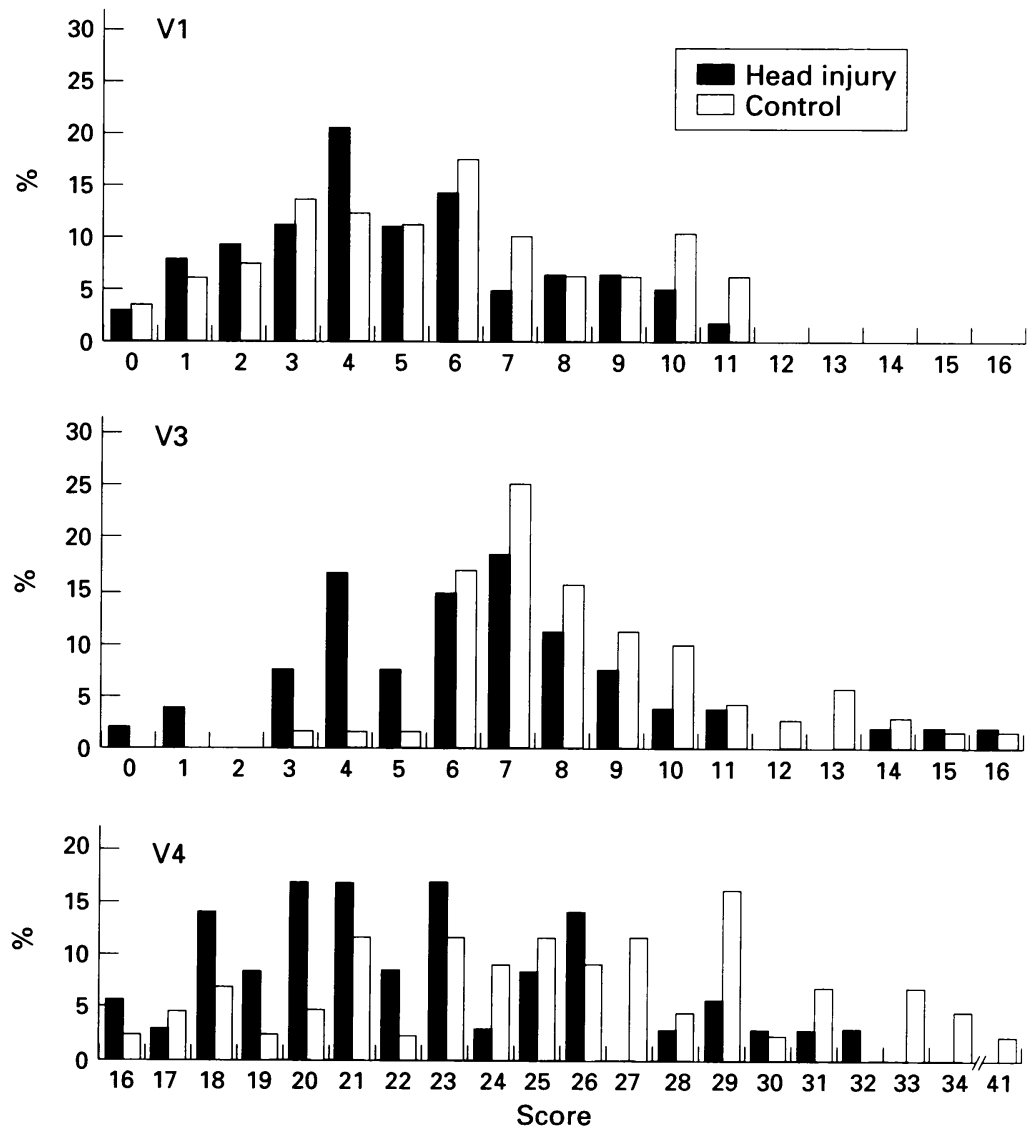

Distribution of visual closure scores after injury, at one year and at age $6 \cdot 5$ years. Head injury = closed columns; controls $=$ open columns; abscissa $=$ visual closure scores Ordinate $=$ percentage of subjects with score; $V 1=$ scores within one month of injury; $V 3=$ scores at one year; $V 4=$ scores at age of 6.5 years compared with the control 3.40 and 3.50 years; this difference was not statistically significant.

Patients lost to inclusion at six and 12 months-Of the 78 children in the head injury group, 69 were seen at six months, six being completely lost to follow up and three were not seen on this one occasion but seen later. Ten had had another head injury, so that their scores were not used in further analysis, leaving 59. Between six and 12 months there had been two further head injuries, leaving 57 cases for analysis. Of the 86 controls seven were completely lost. Two were not seen at six months and seen at 12 , and two were seen at six months and not at 12 . One had a head injury in the first interval and one in the second, leaving 77 for analysis at 12 months.

Clinical history-The parents of HI group children were asked whether their child had been unconscious and if it had cried immediately. In $42 \%$ and $36 \%$ respectively they were unable to say. A definite report of unconsciousness was given in only $5 \%$ and $53 \%$ said the child had not been unconscious. In $38 \%$ the child had cried at once. In $60-70 \%$ the child was described as being drowsy, dazed, or sleepy for a while. Thirty four per cent vomited and $43 \%$ complained of a headache. There was a bruise on the head in $61 \%$. Seventy eight per cent of the injuries were due to falls, $17 \%$ to being struck by objects such as swings, or running into them, and $5 \%$ were due to motor vehicle accidents.

The parents of the control group were asked similar questions; $88 \%$ of the children had cried immediately, none had been unconscious, and some $10 \%$ had been sleepy or subdued, but none had vomited or complained of the other symptoms reported in the HI group.

Cognitive tests-Analyses of variance (ANOVA) were conducted with the neuropsychological data as dependent variables, with independent variables type of injury, age at time of injury, sex, and socioeconomic state.

At the first test, in the first month after injury, there was no difference on any measure between the head injury and the control groups. Six and twelve months later, however, the HI group did less well $(F=20.43$, $\mathrm{P}=0.0001$ and $F=10.61, \mathrm{P}=0.0016)$ than the controls on the visual closure test, which requires the child to find objects concealed in pictures in a limited time. This effect was independent of the slight preponderance of younger children in the HI group. The figure shows the distribution of the scores and the table their means. The mean increase in visual closure scores over 12 months was 1.83 for the HI group and 3.35 for the controls; the difference was significant $(t=-2 \cdot 8777, \mathrm{P}=0.005)$. There was no significant difference between the groups on any other measure, the scores of the HI group and controls being remarkably similar.

Further injury-Ten children of the HI group had another mild head injury in the six months after the first, and three in the second six months (one for the second time), 13.9\% and $4.2 \%$ of those at risk. Two of the control 
group also had a mild head injury, one in the first six months, and one in the second (1.3\%). The difference in incidence between the $\mathrm{HI}$ and control groups in the first six months was significant ( $P=0.0023$ (Fisher's exact test)). In both groups parents reported injuries other than those to the head, five in the HI group and 18 in the control group. The number of injuries of all sorts was therefore similar in the two groups ( $25 \%$ and $25 \cdot 3 \%$ ).

\section{Part 2}

Number of subjects-At the age of 6.5 years complete results were available for 49 children without a second injury in the first year; two of these had mild head injuries subsequently, leaving 47 for analysis. Of 56 controls without head injury in the first year four had a head injury subsequently, leaving 52 for analysis.

Cases lost to follow up at 6.5 years-The means, medians, and ranges of visual closure scores at one year did not differ significantly in the available $\mathrm{HI}$, available control, and lost control groups. The scores were significantly lower in the lost $\mathrm{HI}$ group $(\mathrm{P}=0.005$, median test).

Cognitive tests-Analyses of variance were again conducted with the neuropsychological data as dependent variables, with type of injury, time since the injury, sex, and socioeconomic state as independent variables.

Again the only difference between the HI and control groups was in the visual closure scores $(F=6.19, P=0.015)$, the controls again scoring higher (table and figure). There was no dependence on the time elapsed since injury or on sex.

Tests of reading ability-For the $\mathrm{HI}$ group there were significant (Pearson) correlations between the visual closure scores at six months $(r=0.35, \mathrm{P}<0.05)$ and 12 months $(r=0.45$, $\mathrm{P}<0.01)$ after injury and the reading scores at 6.5 years, low visual closure scores being associated with low reading scores. By contrast, in the control children the visual closure scores did not relate significantly to reading scores. The relation to the visual closure score at 6.5 years was not significant.

Reading recovery had been provided for 13 of the 45 children in the HI group (29\%) and eight of 58 children of the control group $(14 \%)\left(\chi^{2}=3.56, P \approx 0.06\right)$. As the difference is in the direction predicted by the original hypothesis, a one tailed test can be appropriate, with $\mathrm{P}<0.05$.

Behaviour-On the teacher's questionnaire there was no overall difference between the scores of the HI and control groups $(t=1 \cdot 31$, $P>0.05)$. Scores were higher, however, for children who had had reading recovery (means 7.39 and 3.32, $t=3.95, \mathrm{P}<0.01$ ). This was true for the HI and control groups separately, but the difference was greater in the HI group.

\section{Discussion}

The major finding was that in preschool children who had had a mild head injury there was a significant chance that their ability to interpret visual puzzles, expressed as their score on the visual closure test, would be impaired. The impairment was not present immediately after the accident, but was evident six months later, and persisted to the age of 6.5 years. More of the HI group had difficulties with reading in their first year of school and needed special tuition, and within this group the reading ability at 6.5 years was significantly related to the visual closure score six and 12 months after injury.

Some points in the methodology need comment. Strict definition of the severity of the injury presented problems. In most cases the child was not being watched at the time of the accident and often it was difficult for the parent to know whether the child had actually been unconscious, or had cried at once. In the event they were only able to answer this question in some $60 \%$ of cases, and in only $5 \%$ of these were the parents certain that there had been a definite loss of consciousness. This pattern of mild head injury in younger children has been well documented, with a similar incidence of clinical findings. ${ }^{15}$

In fact the range of severity was determined by management decisions. Children were included in the HI group if they had been sufficiently affected by the head injury for their parents to take them to hospital and for a diagnosis of head injury to be made, but not if they then needed to be admitted. Children who had skull fractures or who still had definite symptoms after four hours of observation were therefore excluded.

It would have been satisfying if a further measure of the severity of the head injury had been possible. In adults the duration of posttraumatic amnesia is conventionally used for this purpose, and it is possible to make some estimate of post-traumatic amnesia in even small children. ${ }^{16}$ We have, however, shown in adults that the estimates of post-traumatic amnesia of duration up to an hour are not helpful in grading the severity of mild injury, both because they are unreliable and because they do not relate well to the incidence of cognitive and other problems later. ${ }^{17}$ In the event it was decided that to use this measure would be a fruitless complication.

The children of the control group had injuries which seemed to be very similar in terms of the stresses of treatment needed in the accident department and later. The socioeconomic and development factors were closely comparable, and the children of the two groups performed in the tests in a virtually identical way except for the visual closure scores at the second and subsequent occasions, and for those of reading ability.

Many observations and tests were made on each child, and it could be held that by chance at least one would show a difference between the groups. It was, however, the original hypothesis derived from the pilot study that the anomaly of visual perception and associated reading problems would be found, and the other investigations were accessory to this. The changes in visual closure were seen on three occasions over periods of two to four years, the differences in scores of individual 
patients over time were consistent, and scores on the reading test, an independent measure, were related to visual closure. Under these circumstances it is reasonable to dismiss this statistical objection.

The investigator carrying out the tests knew the history of the child and an expectancy effect could have been introduced. The single investigator was aware of the problem, but an alternative was not possible with the resources available. The limitation has to be acknowledged.

Follow up was incomplete in both parts of the study. This was due to movement of families outside the area, and in only one case to refusal by the parents. The visual closure scores and the socioeconomic state of parents of the lost and remaining cases were compared. There was no significant difference in part 1 . In part 2 the visual closure scores of the lost HI group children were lower than those of the remaining HI group; the scores of the remaining HI group children and of the lost and remaining controls were all similar. Retention of cases with a lower visual closure score would be likely to increase the effect observed, so that their loss does not diminish the relevance of the major findings.

Why was it that the visual closure and no other scores were affected? Why at first did the visual closure scores not differ from controls but later fail to increase normally with age so that there was a persistent deficit?

The Illinois test of psycholinguistic abilities contains several visual tests, but only visual closure was affected. The test presents a line drawing and requires the child to search for one or two objects shown in the margin-for example, a hammer and a saw. These are partly concealed, showing perhaps only the serrated edge of the saw or the head of the hammer, and placed at different angles. The child therefore has to recognise the whole from the part and allow for rotation and differing viewpoints, and also to form a strategy to do this within a limited time. Part of the process would be expected to depend on what in adults would be minor hemispheric temporal lobe function, but an important component may be the planning and execution, which would depend on function of the frontal lobes. Of the other tests that the children did, the one most comparable with visual closure was part 2 of the Frostig developmental test of visual perception, but scores on this did not discriminate between the two groups, perhaps because there is no time limit on this task and it is made easier by allowing the child to outline the figures with coloured pencils.

There is good evidence that in children tests of the "performance" group such as visual closure are more affected by head injury than those of the "verbal" group. . $^{181}$ The reason for this is not clear. Some performance tests are time limited, so that low scores could be due to the slowing of cognitive function that occurs after head injury in children as it does in adults. ${ }^{18}$ This may be a factor in general, but does not apply in the present situation, as there was no impairment of the visual closure scores in the first test shortly after injury, when slowing of function would be expected to be at its peak. Also no difference was found between groups in the visual sequential memory test, which is also time limited.

No brain CT or MRI, were done on the children in this study. Good evidence exists, however, that even in mild head injury MRI shows changes, usually in the temporal and frontal regions and often in both, which relate to neuropsychological impairments. ${ }^{22}$ Although in children of this age hemispheric differences are not well established, if lesions of one temporal lobe were to be the cause of difficulty with visual closure, it would be expected that in a group of cases lesions of the other lobe would occur and that some verbal defects would also be found. This was not the case. This could suggest that the frontal lobe component of the lesions was more likely to be responsible for the changes seen. It certainly seems likely that this is the cause of some facets of cognitive impairment in rather more severe head injuries in childhood. ${ }^{23}$

The selective effect on visual closure could also occur because it depended on a function which was developing particularly rapidly at the time of the accident. Although the development of "verbal skills" probably precedes that of the "performance skills", both are likely to be in active growth in the 2.5 to 6.5 year age range, and it is surprising that there should be sufficient difference in growth rates to produce a relative defect of this size.

The finding that visual closure scores were normal at the first test but then failed to increase at the same rate as controls suggests that it was not an established visual skill that was damaged, but that further development of this skill was impaired, so that the affected children were still not performing as well as controls two to four years later. This pattern of increasing defect is likely to be typical of the developing brain, and is the reverse of that seen in adult neurology when the maximum effect is seen at once and diminishes with time.

Intuitively it was to be expected that defects of visual interpretation would be associated with reduction of reading ability. In the HI group the visual closure scores one year after the injury were related to the reading scores when the children were tested at 6.5 years of age, and the HI group children needed special help with their reading more often than controls. The relation of visual closure at 6.5 years to reading scores was not significant, suggesting that alternative stratagems for reading may have developed, perhaps as a result of the special tuition given to some of the subjects.

There was a relation between the need for special help with reading and general behaviour in school as represented by the score on the teacher's questionnaire. Although this was stronger in the children with head injury, it affected the controls as well. This could be taken to support the contention that children who have accidents belong to a group which can be expected to behave badly, ${ }^{1-3}$ although this is not supported by other authors. ${ }^{25}$ Equally, it may indicate that children who fail 
to achieve find difficulty in social adjustment.

The incidence of further accidents was much the same in both groups. In the first six months, however, in the children in the HI group these resulted in further head injury symptoms. After this they had accidents similar in frequency and resulting symptoms to those of the control group. This resembles our experience with mild head injury in adults, who often find that for a month or two after the injury even a slight blow to their head results in a degree of headache and perhaps drowsiness that would not have been expected. In children parenchymal lesions seen on MRI persist, although reducing in size, for three months or more, ${ }^{22}$ and it is reasonable to postulate that such lesions may make the brain more sensitive to injury.

Despite the positive findings we present here there is little support in other studies of injured children for persisting cognitive impairment specific to mild head injury. Earlier work ascribed continuing problems to pre-existing deficits, behavioural problems, or parental overreaction. ${ }^{1-35}$ In Gulbrandsen's study of children aged 9-13 years, ${ }^{20}$ impairment of performance skills evident at four to eight months after mild injury was not detectable at two years (personal communication, quoted in Levin $e t a^{4}$ ). Bijur and her colleagues carried out a carefully controlled study in which children aged 5 to 9 with mild head injury were compared with children with other injuries and with uninjured children. ${ }^{4}$ Children injured in any way tended more often to be aggressive and hyperactive than uninjured children and to show lower academic achievement, but the head injured differed little from those with other injuries. They concluded that head injury had no discernable effect on general measures of intelligence, achievement, and aggression one to five years after the injury. They did not, however, use a test comparable with the visual closure test and the children were older (only five of 114 subjects were under 6 years old) and may have passed the critical age for the development of some of the skills measured. They did, however, find that the achievement in mathematics at 10 years depended closely $(P=0.006)$ on the age at which the head injury had occurred, scores varying from 93.5 for injuries at 5 years to 104.3 at 9 years of age. The authors did not think that the impairment was a consequence of injury, expecting that the more recent injuries would have more pronounced residual effects, the picture seen in adult neurology. In fact the finding would support the concept that the earlier years are important for developing mathematical skills, and that in younger children the development is more affected by injury.

In conclusion it seems reasonable to say that although in children a mild head injury may not have a long term effect on cognitive function in general, there is good evidence that if it occurs at an age which is critical for the development of a certain skill there may be a persisting deficit. In this way injury in the preschool years seems able to affect the process of learning to read.

As a practical measure, it should be possible for primary school teachers to pay particular attention to children known to have had a head injury, so that help may be given as soon as possible. In more general terms, the possibility should be more widely recognised that mild head injury in children of any age can have subtle effects on school progress and behaviour. Difficulties at school which become evident after a mild head injury are not necessarily or entirely due to pre-existing behavioural traits as has been suggested. Teachers ought to be aware of this and resources for diagnosis and remedial teaching should be made available.

We are much indebted to Dr Philip Voss for statistical advice. The investigation was funded by the New Zealand Neurological Foundation.

1 Rune V. Acute head injuries in children: a retrospective epidemiological, child psychiatric and electroencephalographic study on primary school children in Umeå. Acta Paediatr Scand Suppl 1970, 209.

2 Craft AW, Shaw DW, Cartlidge NEF. Head injuries in children. $B M \mathcal{F} 1972 ; 4: 200-3$.

3 Rutter M, Chadwick O, Shaffer D, Brown G. A prospective study of children with head injuries: I. Design and methstudy of children with head injuries:

4 Bijur PE, Maslum M, Golding J. Cognitive and behavioural sequelae of mild head injury in children. Pediatrics 1990;86:337-44.

5 Casey R, Ludwig S, McCormick MC. Morbidity following minor head trauma in children. Pediatrics 1986;78: 497-502.

6 Gronwall D, Wrightson P. Delayed recovery of intellectua function after minor head injury. Lancet 1974;2:605-9.

7 Wrightson P, Gronwall D. Time off work and symptoms after minor head injury. Injury 1981;12:445-54.

8 Elley WB, Irving JC. Revised socio-economic index for New Zealand. NZ fournal of Educational Studies 1976; 11:25-36.

Doll AE. The measurement of social competence. Minneapolis: Educational Publishers, 1953.

10 Connors C. Connors parent's questionnaire. Psychopharmacol Bull 1973;9(suppl):231-4.

11 Reynell J. Developmental language scales. Windsor: NSER Publishing Co, 1969

12 Kirk SA, McCarthy JJ, Kirk WD. The Illinois test of psycholinguistic abilities. Urbana: University of Illinois Press, 1968.

13 Neale MD. Neale analysis of reading ability (2nd ed). London: Macmillan Education, 1966.

4 Frostig M. Marianne Frostig developmental test of visual perception. 3rd ed. Palo Alto: Consulting Psychologists Press Inc, 1951.

15 Jamison DL, Kaye HH. Accidental head injury in childhood. Arch Dis Child 1974;49:376-81.

16 Ruijs MBM, Keyder A, Gabreëls FJM. Assessment of posttraumatic amnesia in young children. Dev Med Child traumatic amnesia in

17 Gronwall D, Wrightson P. Duration of post-traumatic amnesia after mild head injury. Fournal of Clinical Neuropsychology 1980;2:51-60.

18 Bawden HN, Knights RM, Winogron HW. Speeded performance following head injury in children. $f$ Clin Exp Neuropsychol 1985;7:39-54.

19 Filley CM, Cranberg LD, Alexander MP, Hart EJ. Neurobehavioural outcome after closed head injury in childhood and adolescence. Arch Neurol 1987;44:194-8.

20 Gulbrandsen GB. Neuropsychological sequelae of light head injuries in older children 6 months after trauma. fournal of Clinical Neuropsychology 1984;6:257-68.

21 Levin HS, Eisenberg HM, Wigg NR, Kobayashi K. Memory and intellectual ability after head injury in children and adolescents. Neurosurgery 1982;11:668-73.

22 Levin HS, Williams DH, Eisenberg HM, High WM Jr, Guinto FC Jr. Serial MRI and neurobehavioural findings Guinto FC Jr. Serial MRI and neurobehavioural findings Psychiatry 1992;33:255-62.

23 Levin HS, Culhane KA, Mendelsohn D, et al. Cognition in relation to magnetic resonance imaging in head injured relation to magnetic resonance imaging in head injured

24 Levin HS, Ewing-Cobbs L, Fletcher JM. Neurobehavioural outcome in mild head injury in children. In: Levin HS, outcome in mild head injury in children. In: Levin HS, Eisenberg HM, Benton AL, eds. Mild head

25 Partington MW. The importance of accident proneness in the aetiology of head injuries in childhood. Arch Dis Child 1960;35:215-23. 